\title{
Review
}

\section{Literature review and analysis of the application of health outcome assessment instruments in Chinese medicine}

\author{
Feng-bin Liu ${ }^{1}$, Zheng-kun Hou ${ }^{1}$, Yun-ying Yang ${ }^{1}$, Pei-wu $\mathrm{Li}^{1}$, Qian-wen $\mathrm{Li}^{1}$, Nelson Xie ${ }^{2}$, \\ Jing-wei $\mathrm{Li}^{1}$, Xiang-jing Zeng ${ }^{3}$ \\ 1. Department of Spleen-Stomach Diseases, the First Affiliated Hospital, Guangzhou University of Chinese \\ Medicine, Guangzhou 510405, Guangdong Province, China \\ 2. The School of Medical and Molecular Biosciences, University of Technology Sydney, Ultimo, New South \\ Wales, 2007 Australia \\ 3. Jiangmen Wuyi Traditional Chinese Medicine Hospital, Jiangmen 529000, Guangdong Province, China
}

OBJECTIVE: To evaluate the application of health assessment instruments in Chinese medicine. METHODS: According to a pre-defined search strategy, a comprehensive literature search for all articles published in China National Knowledge Infrastructure databases was conducted. The resulting articles that met the defined inclusion and exclusion criteria were used for analysis.

RESULTS: A total of 97 instruments for health outcome assessment in Chinese medicine have been used in fundamental and theoretical research, and 14 of these were also used in 29 clinical trials that were randomized controlled trials, or descriptive or cross-sectional studies. In 2152 Chinese medicine-based studies that used instruments in their methodology, more than 150 questionnaires were identified. Among the identified questionnaires, 51 were used in more than 10 articles $(0.5 \%)$. Most of these instruments were developed in Western countries and few studies (4\%) used the instrument as the primary evidence for their conclusions.

CONCLUSION: Usage of instruments for health outcome assessment in Chinese medicine is increasing rapidly; however, current limitations include selection rationale, result interpretation and standardization, which must be addressed accordingly.

KEYWORDS: treatment outcome; health-related quality of life; patient-reported outcome; personal satisfaction; questionnaires; instrumentation; medicine, Chinese traditional; outcome assessment (health care)

DOI: 10.3736/jintegrmed2013023

Liu FB, Hou ZK, Yang YY, Li PW, Li QW, Xie N, Li JW, Zeng XJ. Literature review and analysis of the application of health outcome assessment instruments in Chinese medicine. J Integr Med. 2013; 11(3): 157-167.

Received August 27, 2012; accepted December 7, 2012.

Open-access article copyright (C) 2013 Feng-bin Liu et al.

Correspondence: Feng-bin Liu, MD, Professor; Tel: +86-20-36591109; E-mail: liufb163@163.com.

Feng-bin Liu and Zheng-kun Hou contributed equally to this work.

\section{Introduction}

Health assessment instruments in Chinese medicine are developing at a rapid rate ${ }^{[1]}$. These instruments serve as tools that were used by researchers, physicians and patients to measure physical, psychological, and social well-being of the patients. At their core, health assess- ment instruments are the most useful tools in clinical trials and practice. Instrument development is an essential but intermediate step, rather than the ultimate end-goal in instrument-related studies.

Currently in China, many Chinese medicine-specific instruments that were developed by Chinese medical researchers have minimal application in clinical practice, compared with comparable questionnaires developed by 
Western medical researchers, which are common clinical tools. The high volume of Chinese medicine instrument development as compared to low volume of instrument application marks many wasted resources in scientific research, and also raises the question of the validity and reliability of those Chinese medicinal studies that did not utilize health assessment instruments. This study aims to explore and analyze the application of health assessment instruments in Chinese medicine.

\section{Methods}

\subsection{Concepts and terminologies}

"Chinese medicine", "health outcome" and "instrument" were defined in the previous study ${ }^{[1]}$.

\subsection{Search strategies}

\subsubsection{Source, formula and parameters}

Literature searches in China National Knowledge Infrastructure $(\mathrm{CNKI})$ with the formula of “ $(\mathrm{AB}=$ ( 量 + 问 + 调查 $) *($ 表 + 卷 $)+$ 工具 + 条目 $)$ and $((\mathrm{SU}=$ 随即对照 试验 + 对照临床试验 + 临床试验 or $\mathrm{AB}=$ 随机 + 安 慰 + 描述性 + 观察性 + 前瞻性 + 回顾性 or $\mathrm{TI}=$ 随 机 + 安慰 + 试验 $) \operatorname{not}(\mathrm{SU}=$ 动物 not $\mathrm{SU}=$ 动物 * 人 ))" were performed. Subject domain was limited to within the three sub-libraries of "Chinese medicine", "Chinese pharmacology" and "integrated traditional Chinese and Western medicine", while the date range was " 1915 to 2012". To trace the applications of those instruments in clinical trials, we also retrieved full-texts of 97 articles that included health outcome assessment instruments in traditional Chinese medicine, as listed in the previous article ${ }^{[1]}$. The last search was performed on January 20th, 2012.

\subsubsection{Inclusion criteria}

Literature included must have met the following conditions: (1) a study subheading under Chinese medicine research; (2) randomized clinical trials, and descriptive or observational studies that included outcome evaluations.

\subsubsection{Exclusion criteria}

Literature was excluded if the study met any of the following conditions: (1) non-clinical trials, including animal experiments, reviews, systematic reviews, meta-analyses and the others; (2) cross-sectional, diagnostic and prognosis studies without outcome evaluations; (3) updated revision and/or translation of existing instruments; (4) theoretical and educational research on instruments; (5) instruments that were only mentioned in the background or the introduction but were not analyzed in discussion; (6) the title of instruments used in the study was not mentioned and can not be identified; (7) duplicate publications; (8) full-text is unobtainable. It must be noted that the 97 instruments in Chinese medicine were not excluded by (1) and (2) for the purpose of descriptive analyses.

\subsubsection{Data management}

The steps of data management in this study were performed as previously described ${ }^{[1]}$.

\section{Results}

\subsection{Literature search results}

According to the above described procedure, 4557 records were retrieved from the CNKI database. After strict selection with the inclusion and exclusion criteria, 2152 records were identified for this study. More than 60 categories were involved, including clinical medicine of traditional Chinese medicine $(50.1 \%)$, Chinese medicine neurology and psychiatry (21.7\%), internal Chinese medicine (13.6\%), clinical discipline of integrated traditional Chinese and Western medicine (4.5\%). The instruments mainly focused on depression, acupuncture, quality of life (QOL), vascular dementia, cerebral apoplexy, insomnia, lumbar disc herniation, and coronary heart disease. Involved research institutions included Guangzhou University of Chinese Medicine (13.2\%), Chengdu University of Traditional Chinese Medicine (4\%), Heilongjiang University of Chinese Medicine (3.6\%), Guangdong Hospital of Traditional Chinese Medicine (3.1\%), among others. The research involved various funding programs including National Basic Research Program of China (973 Program), National High-tech Research and Development Program (863 Program), State Science and Technology Support Program, and Natural Science Foundation of China (NSFC). The research volume increased significantly every year, with the number of journal articles in the most recent 5 years being 197 (2007), 238 (2008), 333 (2009), 465 (2010) and 502 (2011). The sub-databases included China Academic Journal Network Publishing Database (CAJD, 50.1\%), China Master's Theses Full-text Database (CMFD, 32.1\%), China Doctoral Dissertations Full-text Database (CDFD, 9.7\%) and China Conferences Full-text Database $(8.1 \%)$.

\subsection{Application of domestically-developed instruments in Chinese medicine}

Combining the retrieved electronic copies of the 97 instruments in Chinese medicine with various interviews with experts, we found that many instruments have been used in clinical trials, and basic and theoretical research ${ }^{[2-30]}$.

\subsubsection{Application in clinical trials}

As shown in Table 1, 14 instruments were used in 29 studies, including randomized controlled trials, descriptive studies and cross-sectional studies ${ }^{[2-30]}$.

\subsubsection{Application in basic and theoretical research}

The instruments were mainly used in the following aspects. (1) To provide sources for related research reviews and expand related research discipline. For example, Liu et $a l^{[31]}$ considered that the Chinese Quality of Life (ChQOL) instrument, a domestic general assessment instrument, reflected the characteristics of Chinese medicine and Chinese culture. Those characteristics specified by the 


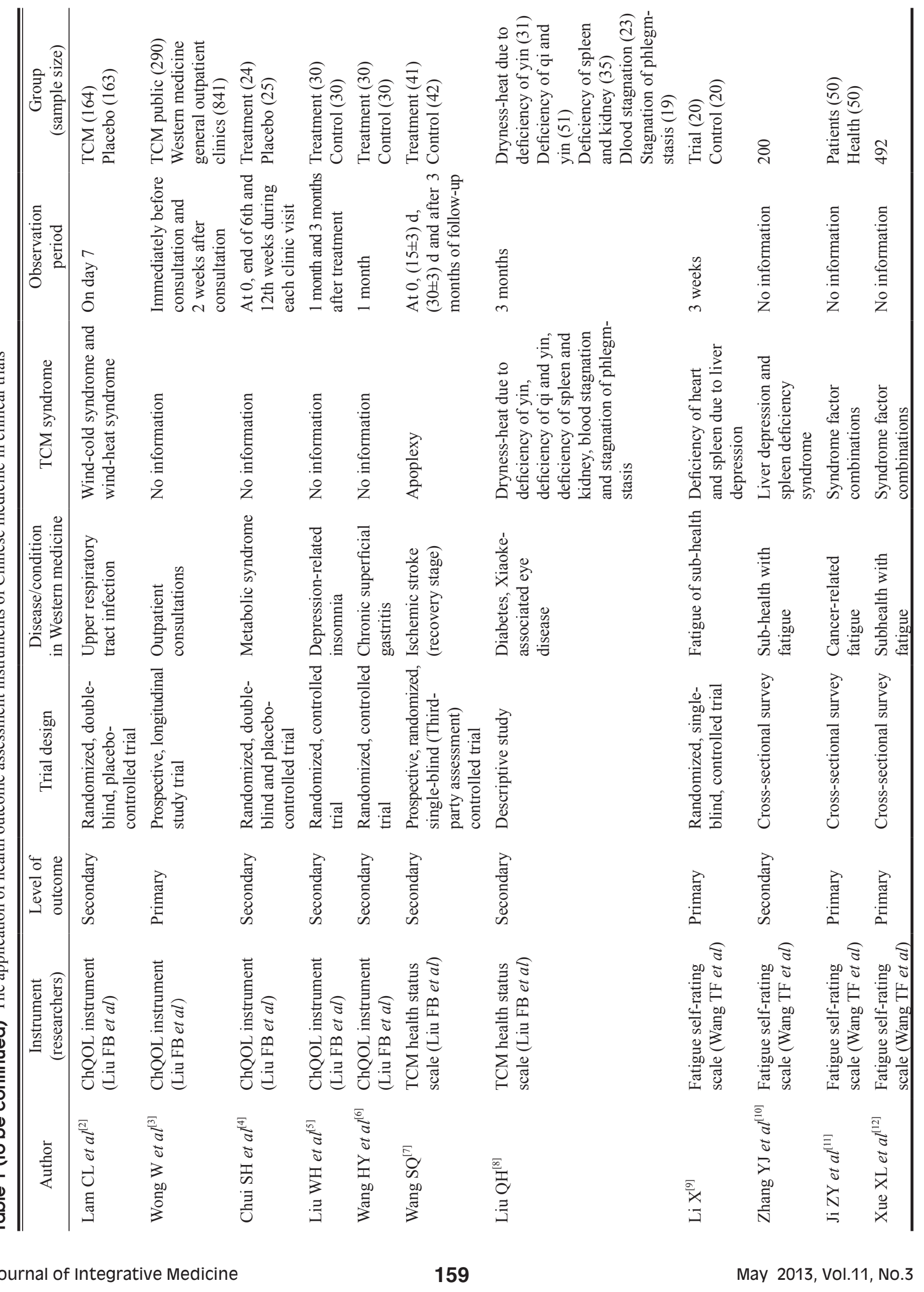




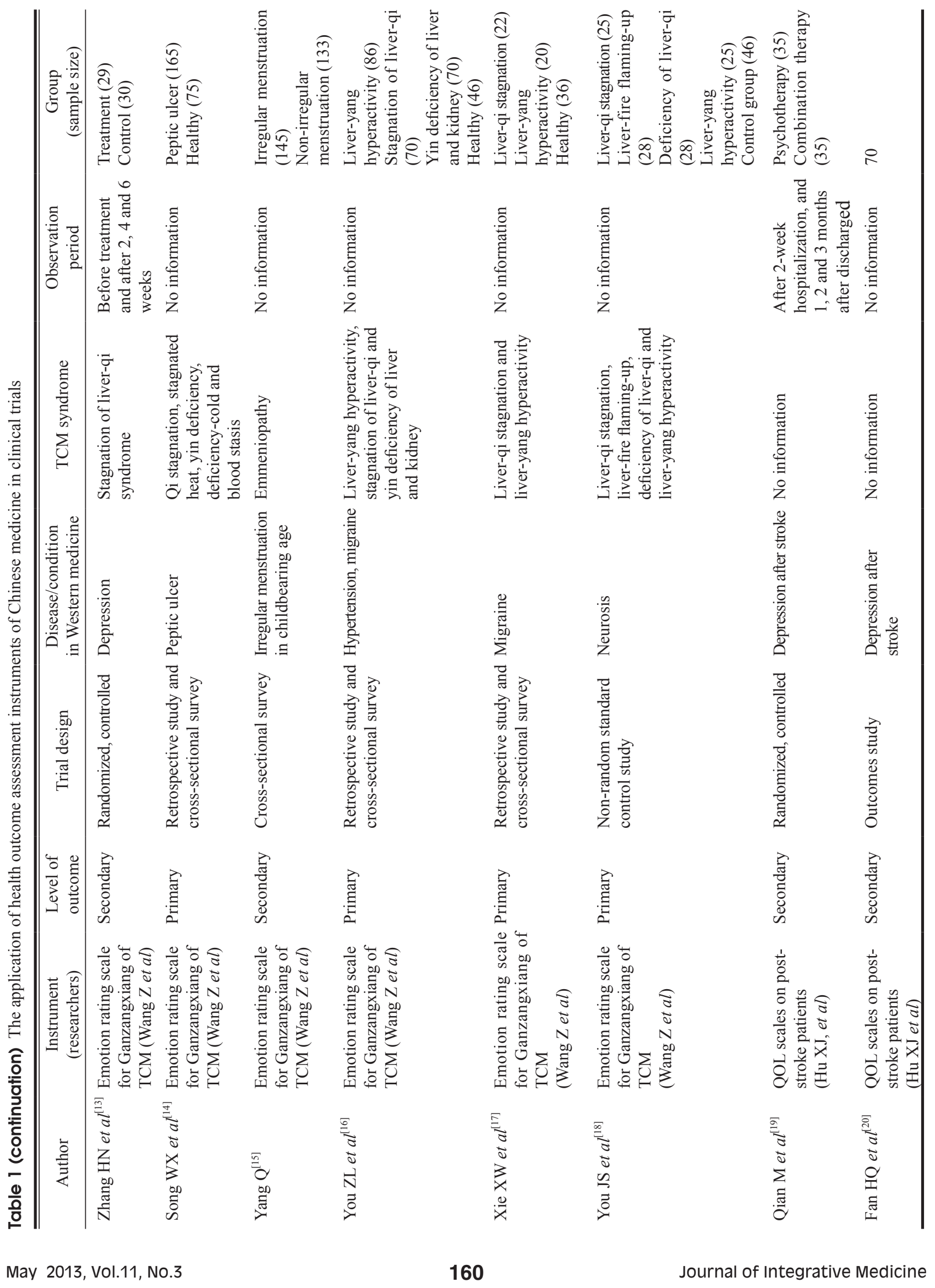




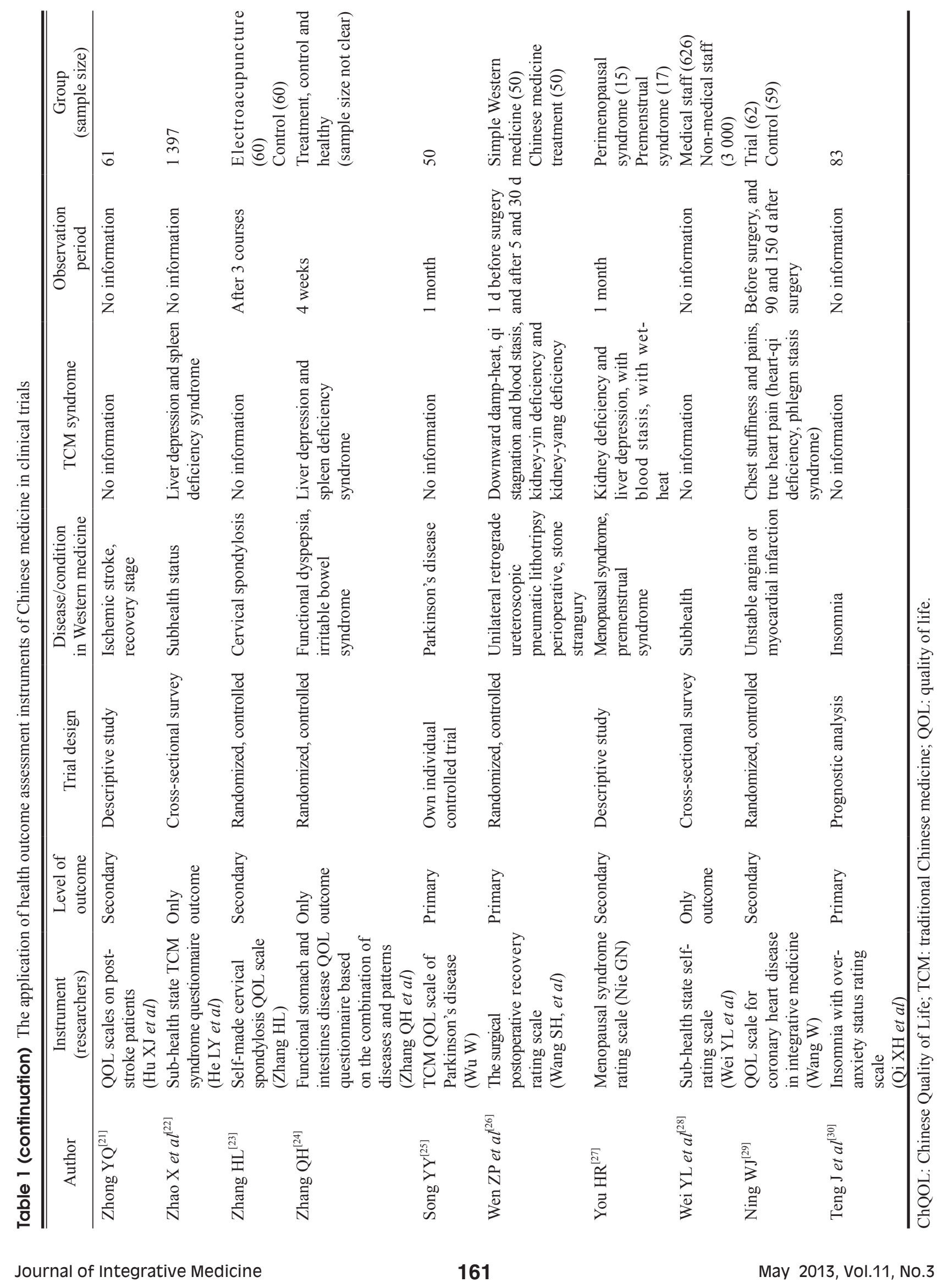


ChQOL borrowed perspective and served as reference for instrument development relevant to Chinese medicine and Chinese culture ${ }^{[32-34]}$. (2) To provide sources for conceptual framework of instrument and item pool development. For example, the Traditional Chinese Medicine PatientReported Outcome Questionnaire for Breast Cancer $^{[35]}$ and Patient-Reported Outcome Efficacy Scale of Traditional Chinese Medicine in Coronary Heart Disease ${ }^{[36]}$ listed the ChQOL as their source of conceptual framework; the Syndrome Element Scale for Diabetic Retinopathy ${ }^{[37]}$ and Scale of Living Quality on HIV/AIDS ${ }^{[38]}$ listed the ChQOL as the source of item pool; the Health Status Scale of Traditional Chinese Medicine ${ }^{[39]}$ served as part of the Evaluation Scale System in Xiaoke-associated Eye Disease for clinical curative effect ${ }^{[8]}$. (3) To provide methodological cues for similar research. For example, the ChQOL instrument provided methods of quality determination that were used by Critical Illness Prognosis Scoring Scale of Traditional Chinese Medicine ${ }^{[40]}$ and Sub-health Symptom Check Scale for College Students ${ }^{[41]}$ for reliability and validity evaluation, and for overall quality evaluation. Quality of Life Scale for Nasopharyngeal Cancer $^{[42]}$ used $t$-test and analysis of variance to measure items, in a similar manner as had been determined by the ChQOL instrument. (4) To provide assessment tools for scientific research. For example, many instruments, such as the Chinese Quality of Life Instrument ${ }^{[31]}$, TCM Syndrome Questionnaire of Sub-health State ${ }^{[43]}$, Emotion Rating Scale for Ganzangxiang of Traditional Chinese Medicine ${ }^{[4]}$ and Fatigue Self-Assessment Scale ${ }^{[45]}$, have been used in clinical research funded by the Research Fund for the Control of Infectious Diseases (Hong Kong SAR Government) ${ }^{[2]}$, 863 Program $^{[20]}$, State Science and Technology Support Program ${ }^{[8,10]}$, National Scientific and Technological Project $^{[12]}$, NSFC ${ }^{[14-16]}$, Specialized Research Fund for the Doctoral Program of Higher Education of China ${ }^{[8,10]}$ and Scientific Research Fund of Hunan Provincial Health Department of Traditional Chinese Medicine ${ }^{[11]}$. (5) To be translated and indexed in international databases. For example, the ChQOL instrument has been translated into an English version ${ }^{[46]}$, Australian English version, Italian version $^{[47]}$ and traditional Chinese version (Hong Kong) ${ }^{[48]}$, and indexed by the Canadian IN-CAM Outcomes Database with full-text download provided.

\subsection{Other instrument application in clinical research of Chinese medicine}

Over 150 instruments were used in 2152 journal articles, including general instruments and disease/conditionspecific instruments. Forty-nine of these instruments were cited by more than 10 studies $(0.5 \%)$. They are as follows (sorted by percentage sizes): Hamilton Depression Rating Scale $(n=397,18.4 \%)$, Mini-mental State Examination $(n=218,10.1 \%)$, Activity of Daily Living Scale $(n=188$, $8.7 \%)$, Various Visual Analogue Scale $(n=162,7.5 \%)$,
Self-rating Depression Scale $(n=145,6.7 \%)$, the Short Form 36 Health Survey (SF-36) $(n=128,5.9 \%)$, Treatment Emergent Symptoms Scale $(n=128,5.9 \%)$, Hamilton Anxiety Scale (HAMA) $(n=113,5.3 \%)$, Self-rating Anxiety Scale $(n=110,5.1 \%)$, McGill Pain Questionnaire $(n=99$, 4.6\%), Pittsburgh Sleep Quality Index $(n=95,4.4 \%)$, Hasegawa Dementia Scale (HDS) $(n=94,4.4 \%)$, Regulated HDS $(n=92,4.3 \%)$, Ashworth Scale $(n=60,2.8 \%)$, National Institutes of Health Stroke Scale $(n=56,2.6 \%)$, Barthel Index $(n=52,2.4 \%)$, Clinical Global Impression Scale $(n=44,2.0 \%)$, Symptom Checklist $90(n=40,1.9 \%)$, the World Health Organization Quality of Life (WHOQOL)BREF ( $n=37,1.7 \%)$, Fatigue Scale-14 ( $n=34,1.6 \%)$, Western Ontario and McMaster Universities Osteoarthritis Index ( $n=29,1.3 \%)$, Fugl-Meyer Assessment $(n=29$, $1.3 \%)$, Rating Scale for Side Effects $(n=29,1.3 \%)$, Blessed Behavioral Scale $(n=26,1.2 \%)$, Neck Pain Questionnaire $(n=23,1.1 \%)$, Functional Activities Questionnaire $(n=23,1.1 \%)$, House Brackmann Grading Scale $(n=22,1.0 \%)$, Athens Insomnia Scale $(n=20,1.0 \%)$, Functional Assessment of Cancer Therapy (FACT) Series Scales $(n=19,0.8 \%)$, Scandinavian Stroke Scale $(n=18$, $0.8 \%)$, Brief Psychiatric Rating Scale ( $n=16,0.8 \%)$, Neurological Deficit Scale $(n=14,0.7 \%)$, Chinese Stroke Scale $(n=14,0.7 \%)$, Modified Barthel Index $(n=14,0.7 \%)$, Clinical Memory Scale $(n=13,0.6 \%)$, Montreal Cognitive Scale $(n=13,0.6 \%)$, Yale Global Tie Severity Scale $(n=13$, $0.6 \%)$, Gross Motor Function Measure $(n=13,0.6 \%)$, Simplified McGill Pain Questionnaire $(n=12,0.6 \%)$, Clinician-administered Scale for DSM-IV $(n=12,0.6 \%)$, Karnofsky Performance Scale (KPS) $(n=12,0.6 \%)$, Seattle Angina Questionnaire $(n=12,0.6 \%)$, Cervical Vertigo Symptoms and Functional Assessment Scales $(n=12$, $0.6 \%)$, Sleep Dysfunction Rating Scale $(n=11,0.5 \%)$, Clinic Spasticity Index $(n=11,0.5 \%)$, Numerical Rating Scale $(n=11,0.5 \%)$, Wechsler Memory Scale $(n=10$, $0.5 \%)$, the Spiegel Score $(n=10,0.5 \%)$ and GESELL $(n=10,0.5 \%)$. In addition, 53 instruments were used in 2 to 9 studies. The remaining instruments were used by only one study. The analyses above did not include the 97 domestic instruments in Chinese medicine.

However, most of these instruments mentioned above were developed in Western settings, only a few of them were domestically developed, such as the Chinese Quality of Life Instrument for Cancer ${ }^{[49]}$. Only a few of studies such as the Rhinoconjunctivitis Quality of Life Questionnaire mentioned obtaining rights for accessing the original instrument ${ }^{[50]}$. Although the data of the vast majority of instruments were appropriately analyzed, many selfdeveloped instruments did not perform a property assessment, such as the Postpartum Prolactin Self-protection Capabilities Assessment Scale ${ }^{[51]}$ and Post Stroke Depression Treated with Acupuncture Assessment Scale ${ }^{[52]}$. Moreover, many instruments were used for outcome assessment; only a 
minority (about 4\%) were used as primary outcomes in the results ${ }^{[53]}$.

\section{Discussion}

\subsection{Characteristics of instrument application}

Chinese medicine instruments have the following characteristics in clinical application. First, health assessment instruments in Chinese medicine have been applied in numerous novel ways, in both clinical and scientific settings. These questionnaires were used in studies with various designs, including cross-sectional survey, descriptive study and prognosis research; they covered internal, surgical and gynecological diseases, acute and chronic diseases, organic and functional diseases, or other pathological conditions. Second, some research was performed without language or regional barriers. International collaborations in instrument research have been increasing rapidly ${ }^{[2,34,54]}$. Third, application of Chinese medicine instruments is increasing yearly, which reflects the trend of Chinese medicine researchers' increasingly open and active role in verifying therapeutic effects of traditional Chinese medicine. Lastly, although over 150 different instruments were used in Chinese medicine clinical trials, a few instruments such as WHOQOL-BREF, HAMA, SF-36 and KPS had much more widespread usage. This shows that the main research directions are fairly stable.

\subsection{Limitations of instrument application}

Some limitations still exist in instrument application. (1) Lack of rationale for instrument selection. Only a few researchers described the reasons for instrument selection which is a crucial step in application research since inappropriate instrument selection may lead to falsepositive or false-negative results, or may unnecessarily increase research burden. (2) Design defects. Most of the clinical trials were poorly designed. For example, many randomized controlled trials did not clearly describe the randomization, allocation concealment, blinding method, and comparison and placebos. The methodology and reporting were poorly performed, and consequently had unreliable results. (3) Unclear outcome selection. Clinical outcomes should reflect the efficacy and safety of interventions, but many selected outcomes in the studies could not achieve this aim. Moreover, many researchers used instruments as secondary outcomes, and preferred foreign instruments without explanations on endpoint model and instrument equivalence. (4) Poor result explanations. Few instruments in Chinese medicine established a normal model for clinical practice and result explanations. Researchers could only estimate their intervention according to the changes in the scores before and after treatment, but could not clearly explain cross-sectional scores, which limited the application of the instruments. (5) Low-level standardization. Very few studies included sufficient details or reached standards in their reporting. Some published studies have outlined appropriate ways to report instrument applications. Although no official standards yet exist, many details which have already been covered in the current research can provide necessary parameters on future instrument research. (6) Unrelated instrument development and application. Currently, many Chinese medicine clinical trials are using instruments developed in other countries, for example, from Western cultures, rather than those developed under the specific theoretical framework of traditional Chinese medicine, which would be a lot more applicable and relevant to Chinese medicine clinical trials. This is an inefficient use of scientific resources.

\subsection{Causes of limitations}

Limitations of instrument application research in Chinese medicine mentioned above may be due to the following reasons. (1) Lack of guidance from academic organizations and support from the government. Currently, many excellent foreign academic organizations and government sectors are involved in health outcome research, including the International Society for Quality of Life Research (ISOQOL), European Regulatory Issues on Quality of Life Assessment Group, Patient-Reported Outcomes Harmonization Group, Mapi Research Institute and the US Food and Drug Administration. Their financial and scientific assets greatly propel the research progress of instrument development; there is a constant, consistent stream of exploration and publication of new findings. In contrast, similar institutions do not exist yet in China. (2) Lack of independent and rigorous research guidance. Although many global guidelines have been developed, most of them were established on the premises of Western culture and their own clinical and research practices. Compared with Western medicine, Chinese medicine clinical trials have some distinct characteristics, so blindly replicating Western research specifications may not provide accurate or complete results. (3) Lack of scientific data and media platform. This kind of platform would integrate academic resources, greatly facilitate the retrieval and judgment of the researchers or patients, and promote strong professional research development. PubMed, Patient-Reported Outcome and Quality of Life Instruments Database, Quality of Life Research, etc., have good operability in practical applications. At present, all domestic databases have different literature sources and retrieval features, which are inconvenient to access, and to a certain extent, hinder communication. Therefore, a national data platform is urgently needed. (4) Poor communication with the public result in undesirable numbers in target populations. Not all patients, clinicians or even researchers clearly understand which instruments can best reflect the target health status, hence instrument usage does not reach its full potential. (5) Lack of multi- 
center cooperation and collaborations under different administrative regions, cultural backgrounds, languages, medical institution levels and disciplines.

\subsection{Countermeasures for limitations}

To solve these problems, the research groups and many instrument experts, clinicians and statisticians together have made many changes. In May 2008, a group of 13 ISOQOL members from Mainland China, Hong Kong and Taiwan established the ISOQOL-Asian Chinese Chapter (ISOQOL-ACC) in Guangzhou, China. Its aim was to promote sharing, collaboration, and education among QOL researchers in the Chinese speaking regions, to encourage discussion and studies on Chinese culture and language-specific issues related to QOL researchers, and to connect QOL researchers in the Chinese speaking regions to the rest of the world ${ }^{[55]}$. The research group has held many conferences and training courses. As of this date, five national QOL academic conferences and three ISOQOL-ACC symposiums were held in the years 2000, 2002, 2004, 2008, 2010 and 2012. These academic conferences focused on instrument development and cross-cultural adaptation, methodology, clinical trials design, analysis and reporting, outcome assessment, Chinese medicine and Chinese culture. After several rounds of expert discussions, inclusion of the latest research findings both domestically and abroad, and several amendments, the draft of Guidelines for Medical Instrument Development and Application ${ }^{[56]}$ was published in 2010. The guidelines detailed several aspects, such as the concept and classification of instruments, rationale, developments, applications, clinical practice, cross-cultural adaptation and statistical analysis. It also illustrated the development and evaluation processes of ChQOL instrument and a Chinese version of WHOQOL. The Preferred Reporting Items for Patient-Reported Outcome Instrument Development (PRIPROID) has been preliminarily drafted ${ }^{[57]}$. PRIPROID, including 23 items of 7 domains, was proposed to provide reference for instrument development reporting. Essentially, a Chinese medicine instrument is a representation of Chinese culture and should not be limited by different medical systems in practice. Thus, we recommend that the Chinese instrument research should abandon differences between Chinese and Western medicine and specificities of various regions, and instead further explore instrument development that is suitable across different cultural and medical systems ${ }^{[58]}$.

However, these efforts are still inadequate for the ideal scenario of further research development. Much more support, collaboration and communication is still needed, which may be achieved by: (1) strengthening national academic cooperation by establishing academic sub-groups, for example, on methodology and clinical medicine, and actively introducing new international academic notions, so that rigorous critical academic thinking may be better practiced among researchers of QOL; (2) establishing an integrated scientific research data and media platform on instrument research to provide a good venue for data integration and information dissemination; (3) seeking more government support in promoting popularization, education and application of instrument research; (4) enhancing theoretical and fundamental research with clear definitions on basic concepts and terminology to standardize instrument research; (5) improving studies on cross-medicinal and cross-cultural instruments and the state and role of Chinese medicine instruments; (6) more communication and cooperation with statistics, psychology, sociology and other disciplines, so that new ideas, methods and ways of thinking can be introduced into the field of instrument research.

\subsection{Advantages, limitations and value of this study \\ 4.5.1 Advantages}

We analyzed Chinese medicine instrument development and application research with independent search strategies. The study procedure was strictly controlled, and included data extraction and analyses. Analyses were prudently and rigorously performed. Thus, we believe our conclusions are logical and reasonably reliable.

\subsubsection{Limitations}

Some limitations exist in our research. (1) The search strategies were not completely comprehensive. First, we limited our search to Chinese medicine, integrated traditional Chinese and Western medicine, Chinese pharmacology sub-categories or a CLC number of R2 (Chinese Library Classification: Chinese medicine) in CNKI. The literature included may not have reflected all progress in academic research. Second, being limited by the classification of each database, the domain definition may have excluded some studies in Chinese medicine, which may have left some Chinese medicine instruments unidentified. In the research of instrument application, the literature was only sourced from CNKI, which again may have left out important information because CNKI does not collects all published documents. (2) Due to space limitations, much information collected by data extraction table was not listed, which may affect the quality evaluation of the conclusion.

However, we believe that these limitations did not impact our conclusions in any significant manner. First, the databases mentioned above have included the vast majority of eligible journals and all the core journals in Chinese medicine, thus they should at least truly reflect current main trends of instrument research in Chinese medicine. Second, although some Chinese medicine research was not included, supplementary searches found that the most of the excluded research was overlapped in the retrieval results and no high-quality instrument was missing. Retrieving results of instrument application research in a single database may have narrowed the 
number of literature results. However, the 2152 filtered records used in this study were independently screened from the original 4557 records by two researchers. This should therefore increase the likelihood that the search result was credible and typical. Last but not least, although some of the data were not listed, vital information for assessment instrument development and application have been provided, so readers can easily track down the results themselves by contacting our research group.

\subsubsection{Value of this study}

Being of a theoretical nature, the clinical value of this study is limited. Our main purpose is to provide a list of assessment instruments to choose from and recommend options that clinicians can use to expand their horizons while evaluating outcomes. The theoretical significance of this study is to systematically display the current situation of development and application of QOL assessment instruments in Chinese medicine for the very first time, from which researchers can understand, analyze and evaluate domesticrelated research. We also analyzed the current limitations in instrument research and the countermeasures that can provide the basis for generating sustainable development strategies of assessment instrument research.

\section{Conclusions}

Due to the complexity in details of assessment instrument development and application, and unavoidable subjective factors during the process of retrieval and screening, the results of this article may not completely represent the current state of domestic assessment instrument development and application; further studies with more rigorous methodologies are needed. At present, a limited amount of data show that domestic research in the development and application of assessment instruments in Chinese medicine are developing rapidly. However, there are still shortcomings in the domains of conceptual framework, study process, attribute evaluation, statistics, application basis, study designation, result analysis and standardization, etc. These limitations are affected by various factors and resolving them will be a complex, long-term process requiring interdisciplinary efforts between different academic, media, and governmental organizations, and even across different nations. Many excellent researchers and clinicians have already made significant contributions in this field. Research on the QOL assessment instruments in Chinese medicine will play an important role in the never-ending mission to improve human health.

\section{Funding}

This research was supported by the National Natural Science Foundation of China (No. 81073163)

\section{Competing interests}

The authors declare that they have no competing interests.

\section{REFERENCES}

1 Liu FB, Hou ZK, Yang YY, Zhang ZZ, Li PW, Zhuang KH. Literature review and analysis of the development of health outcomes assessment instruments in Chinese medicine. J Integr Med. 2013; 11(2): 80-89.

2 Lam CL, Wong W, Fong DY. Chinese herbal medicine in the treatment of acute upper respiratory tract infection: a randomised, double blind, placebo-controlled clinical trial. Hong Kong Med J. 2009; 15 (Suppl 6): 30-34.

3 Wong W, Lam LK, Li R, Ho SH, Fai LK, Li Z. A comparison of the effectiveness between Western medicine and Chinese medicine outpatient consultations in primary care. Complement Ther Med. 2011; 19(5): 264-275.

4 Chui SH, Chow FC, Chan K, Fu S, Szeto YT. Slimpid may boost plasma IGF-1 level and improve the quality of life in patients with risk of developing metabolic syndrome. $\mathrm{J}$ Complement Integr Med. 2008; 5(1): 17.

5 Liu WH, Zhao CY, Lun X, Yu J. Clinical effects of "3 acupoints for sleep" on patients with depression-related insomnia: a report of 30 cases. Zhen Jiu Lin Chuang Za Zhi. 2009; 25(4): 5-6. Chinese.

6 Wang HY, Liu FB. Clinical effects of Chinese herbal medicine modified Sini Powder in treating 30 patients with chronic superficial gastritis and the quality of life evaluation. Jiangsu Zhong Yi Yao. 2011; 43(6): 21-22. Chinese.

7 Wang SQ. Comprehensive evaluation of efficacy of acupuncture in post-stroke patients. Guangzhou: Guangzhou University of Chinese Medicine. 2011. Chinese with abstract in English.

8 Liu QH. The development and preliminary application of evaluation scale system in Xiaoke-associated eye diseases for clinical curative effect. Guangzhou: Guangzhou University of Chinese Medicine. 2007. Chinese with abstract in English.

9 Li X. Clinical research on efficacy of anti-fatigue No. 2 Formula in treating chronic fatigue due to liver stagnation with heart and spleen deficiency. Beijing: China Academy of Chinese Medical Sciences. 2009. Chinese with abstract in English.

10 Zhang YJ, Xue XL, Wang TF, Wang JJ, Li GR, Han P, Zhong YY, Li L, Wang QB, Tang Y, Li Z, Wang WP. Correlation between fatigue and life quality in 200 cases with sub-health with fatigue and liver-qi stagnation and spleen-qi deficiency. Tianjin Zhong Yi Yao. 2010; 27(5): 369-371. Chinese with abstract in English.

11 Ji ZY, Xu YM, Wang XM. Clinical investigation on fatigue characteristics and syndrome differentiation of TCM in patients with CRF. Xian Dai Zhong Liu Yi Xue. 2010; 18(12): 2473-2478. Chinese with abstract in English.

12 Xue XL, Wang TF, Han P, Wang JJ, Li GR, Zhang YJ Study on fatigue characteristics in people with fatiguepredominant sub-health based on different TCM syndromes. Tianjin Zhong Yi Yao. 2011; 28(4): 284-287. Chinese with abstract in English.

13 Zhang HN, Hu SY, Li YH, Zhang CH. Clinical observation 
of Baisong tablets for depression. Hunan Zhong Yi Yao Da Xue Xue Bao. 2008; 28(4): 48-49, 56. Chinese with abstract in English.

14 Song WX, Huang FY, Wang Z. Analysis of emotional state in peptic ulcer with syndromes of traditional Chinese medicine. Zhong Yi Yao Xue Kan. 2006; 24(7): 1236-1237. Chinese with abstract in English.

15 Yang Q. Clinical research on emotion state of women in child-bearing age with abnormal menstruation. Chengdu: Chengdu University of Traditional Chinese Medicine. 2008. Chinese with abstract in English.

16 You ZL, Xie XW, You H. Comparative study on emotional characteristic measurement between hyperhepatic yang syndrome and correlative syndrome. Hunan Zhong Yi Xue Yuan Xue Bao. 2005; 25(5): 1-4. Chinese with abstract in English.

17 Xie XW, Tang FY, You JS, Chen CH. Assessment and analysis on emotion state of patients with migraine due to two liver syndromes of traditional Chinese medicine. Shandong Zhong Yi Yao Da Xue Xue Bao. 2004; 28(6): 427-428. Chinese.

18 You JS, Hu SY, Zhang HG. Assessment and analysis of emotion state of four liver syndromes of traditional Chinese medicine in neurosis patients. Zhongguo Lin Chuang Kang Fu. 2004; 8(24): 5058-5059. Chinese with abstract in English.

19 Qian M, Fang HQ, Niu ZP, Sun MZ. Evaluation of therapeutic effects of psychotherapy alone and combined with prozac on post-stroke depression. Zhong Xi Yi Jie He Xin Nao Xue Guan Bing Za Zhi. 2010; 8(6): 681-682. Chinese with abstract in English.

20 Fan HQ, Qian M, Sun MZ. Study on the quality of life of post-stroke depression patients. Shanxi Yi Ke Da Xue Xue Bao. 2011; 42(3): 188-190. Chinese with abstract in English.

21 Zhong YQ. Research of quality of life scales on poststroke patients treated by traditional Chinese medicine. Guangzhou: Guangzhou University of Chinese Medicine. 2011. Chinese with abstract in English.

22 Zhao X, Chen JX, Wang LM, Cui HT, Cui HZ. Study on common signs and symptoms of liver depression and spleen deficiency syndrome in sub-health. Shandong Zhong Yi Yao Da Xue Xue Bao. 2011; 35(3): 278-280. Chinese.

23 Zhang HL. The clinical and experimental research on electroacupuncture for cervical spondylosis. Guangzhou: Guangzhou University of Chinese Medicine. 2000. Chinese with abstract in English.

24 Zhang QH. Application of functional stomach and intestines disease quality of life scales based on the combination of diseases and patterns. Zhonghua Zhong Yi Yao Xue Kan. 2009; 27(5): 1064-1065. Chinese.

25 Song YY. Preliminary discussion on the evaluation capacity of traditional Chinese medicine quality of life scale applied to evaluate the curative effect of Chinese medicine on Parkinson's disease. Guangzhou: Guangzhou University of Chinese Medicine. 2011. Chinese with abstract in English.

26 Wen ZP, Hong ZM, Lin F, Deng YY, Chen ZF, Yu SL, Wang Y. Observation of the recovery situation after Chinese medicine treatment involved in unilateral retrograde ureteroscopic pneumatic lithotripsy perioperative. Dang Dai Yi Xue. 2009; 15(19): 27-29. Chinese with abstract in English.

27 You HR. Clinical study of comprehensive Chinese medicine treatment (mental and body treatment) in perimenopausal syndrome and premenstrual syndrome. Guangzhou: Guangzhou University of Chinese Medicine. 2011. Chinese with abstract in English.

28 Wei YL, Kong J, Liu GL, Huang Y, Han B, Li L, Chen HM, Kong J, Liu WH, Wei BH. Application of somatic complaints of sub-health status questionnaire (Sc-SHs-Q) in sub-health status's clinical trail screen, symptoms analysis and evaluation of intervention therapy. Huan Qiu Zhong Yi Yao. 2009; 2(5): 349-353. Chinese with abstract in English.

29 Ning WJ. Clinic study on effects of integrated traditional Chinese and Western medicine method on quality of life of patients after PCI. Guangzhou: Guangzhou University of Chinese Medicine. 2011. Chinese with abstract in English.

30 Teng J, Qi XH, Kang XL, Wang L. Insomnia with overanxiety status and risk factor analysis. Shandong Zhong Yi Yao Da Xue Xue Bao. 2010; 34(1): 19-20, 22. Chinese.

31 Liu FB, Zhao L, Lang JY, Lin LZ, Liang GH, Fang JQ. Development of the Chinese quality of life instrument. Zhongguo Zu Zhi Gong Cheng Yan Jiu Yu Lin Chuang Kang Fu. 2007; 11(52): 10492-10495, 10515. Chinese with abstract in English.

32 Li QZ. National Chinese Medicine Clinical Research Center: How to develop the research roadmaps for key diseases. China News of Traditional Chinese Medicine. 2010-8-11 (003). Chinese.

33 Wong W, Lam CLK, Shan JST, Fong DYT. Effectiveness of traditional Chinese medicine in primary care. In: Kuang HX. Recent advances in theories and practice of Chinese medicine. InTech. 2012: 187-208. (2012-01-28) [201207-25]. http://www.intechopen.com/source/pdfs/26164/ InTech-Effectiveness_of_traditional_chinese_medicine_in primary_care.pdf.

34 Sertel S, Greten HJ, Kraeme H Jr, Efferth T, Plinkert PK, Baumann I. Evaluation of quality of life in patients treated with traditional Chinese medicine. Open Otorhinolaryngol J. 2010; 4: 62-67.

35 Zhang J, Shen Y, Bian WH, Pan LQ. Research on the design of primary TCM PRO questionnaire for breast cancer. Nanjing Zhong Yi Yao Da Xue Xue Bao. 2011; 27(3): 295297. Chinese with abstract in English.

36 He QY, Wang J, Shi Z, Xiong XJ. The conception of theoretical structure of PRO efficacy scale of traditional Chinese medicine in coronary heart disease. Zhonghua Zhong Yi Yao Za Zhi. 2010; 25(1): 42-45. Chinese with abstract in English.

37 Jiang XF. Development and application of a syndrome element scale for diabetic retinopathy. Chengdu: Chengdu University of Traditional Chinese Medicine. 2009. Chinese with abstract in English.

38 Zhang ML, Wei JY, Wu YM, Guo XX, Su FJ. Preparing scale of living quality on HIV/AIDS (HIV/AIDSQOL-46). Zhong Yi Xue Bao. 2009; 24(5): 6-8. Chinese with abstract in English.

39 Liu FB, Lang JY, Zhao L, Fang JQ, Liang GH, Lin LZ. The development of health status scale of traditional Chinese medicine (TCM-HSS). Proceedings of the 19th National Academic Seminar on Spleen-gastric Diseases, Branch of Spleen-gastric Diseases of China Association of Chinese Medicine. Shijiazhuang, China. 2007: 589-593. Chinese.

40 Li J. Preliminary research on establishing critical illness 
prognosis scoring scale of traditional Chinese medicine. Guangzhou: Guangzhou University of Chinese Medicine. 2009. Chinese with abstract in English.

41 Yang S, Chen C, Liu XJ, Yang TB. Reliability and validity of a sub-health symptom check scale for college students. Wei Sheng Yan Jiu. 2010; 39(4): 491-493. Chinese with abstract in English.

42 Guo LF. The preliminary development of quality of life scale for nasopharyngeal cancer. Guangzhou: Guangzhou University of Chinese Medicine. 2008. Chinese with abstract in English.

43 He LY, Liu BY, Xie YM, Hu JQ, Li WQ, Gao Y. Evaluation of traditional Chinese medicine syndrome questionnaire for sub-health state. Zhongguo Zhong Yi Ji Chu Yi Xue Za Zhi. 2004; 10(10): 64-67. Chinese.

44 Wang Z, Hu SY, Cai TS, Xia DS. Development of emotion rating scale for Ganzangxiang of traditional Chinese medicine (ERSG). Zhongguo Xing Wei Yi Xue Ke Xue. 2004; 13(1): 104-106. Chinese with abstract in English.

45 Wang TF, Xue XL, Ji SL. Imaging for the development of fatigue self-assessment scale. Proceedings of the Conference on the Establishment of the Diagnostics Branch of China Association of Chinese Medicine, Beijing, China. 2006: 250-253. Chinese.

46 Leung KF, Liu FB, Zhao L, Fang JQ, Chan K, Lin LZ. Development and validation of the Chinese quality of life instrument. Health Qual Life Outcomes. 2005; 3: 26.

47 Aschero G, Fenoglio F, Vidili MG, Wussler A. The ChQoL questionnaire: an Italian translation with preliminary psychometric results for female oncological patients. Health Qual Life Outcomes. 2010; 8: 106.

48 Wong W, Lam CL, Leung KF, Zhao L. Is the content of the Chinese Quality of Life Instrument (ChQOL) really valid in the context of traditional Chinese medicine in Hong Kong? Complement Ther Med. 2009; 17(1): 29-36.

49 Xiong L, Cheng ZQ, Zhu SJ, Zhu WT. Observation on efficacy of Xihuang Pill for 18 malignancies patients with metastatic bone pain. Proceedings of the Research on Clinical Applications of Xihuang Pill, Beijing, China. 2009: 52-54. Chinese.
50 Luo QL. Clinical application and effectiveness evaluation of Chinese version rhinoconjunctivitis quality of life questionnaire. Guangzhou: Guangzhou University of Chinese Medicine. 2008. Chinese with abstract in English.

51 Kong FX, Shao LY, Qian F. The effect of TCM dialectical nursing approach on the self-care ability in postpartum women with hypogalactia. Zhonghua Hu Li Za Zhi. 2009; 44(6): 551-553. Chinese with abstract in English.

52 Li F. Clinical observation on post-stroke depression treated by acupuncture combined with fluoxetine. Wuhan: Hubei University of Chinese Medicine. 2009. Chinese with abstract in English.

53 You J, Shi ZM, Han BH, Ding JF. Evaluation on effect of Feiji recipe on quality of life of patients with non-small cell lung cancer by adopting international questionnaire of QOL. Zhongguo Zhong Xi Yi Jie He Za Zhi. 2006; 26(1): 33-37. Chinese with abstract in English.

54 Wu D, Lai S, Zhou L, Guo X, Liang W, Wen Z, Ou A, Zhang G, Chen K. Further validation of the health scale of traditional Chinese medicine (HSTCM). Chin Med. 2009; 4: 8 .

55 Executive Committee, International Society for Quality of Life Research (ISOQOL). Establishment of International Society for Quality of Life Research (ISOQOL). Proceedings of Symposium of Quality of Life Research for Asian Chinese. Guangzhou, China. 2008: 88-97. Chinese and English.

56 Fang JQ, Liu FB, Hao YT, Liu BY, Xie YM. Guidance for medical instrument development and application (draft). Proceedings of Research on Five-organ Relation Theory and Practice and the Development Methods and Application of Chinese Medicine Questionnaire, Guangzhou, China. 2010: 152-194. Chinese.

57 Hou ZK, Liu FB, Fang JQ, Li XY, Li LJ, Lin CH. Advising on preferred reporting items for patient-reported outcome instrument development: the PRIPROID. Chin J Integr Med. 2013; 19(3): 172-181.

58 Hou ZK, Liu FB, Liang YY, Zhuang KH, Lin CH, Li LJ. On the necessity of developing quality of life instruments in traditional Chinese medicine. J Chin Integr Med. 2011; 9(5): 468-482. Chinese with abstract in English.

\section{Submission Guide}

Journal of Integrative Medicine (JIM) is a PubMed-indexed, peer-reviewed, open-access journal, publishing papers on all aspects of integrative medicine, such as acupuncture and traditional Chinese medicine, Ayurvedic medicine, herbal medicine, homeopathy, nutrition, chiropractic, mind-body medicine, Taichi, Qigong, meditation, and any other modalities of complementary and alternative medicine (CAM). Article types include reviews, systematic reviews and meta-analyses, randomized controlled and pragmatic trials, translational and patient-centered effectiveness outcome studies, case series and reports, clinical trial protocols, preclinical and basic science studies, papers on methodology and CAM history or education, editorials, global views, commentaries, short communications, book reviews, conference proceedings, and letters to the editor.

\section{- No submission and page charges • Quick decision and online first publication}

For information on manuscript preparation and submission, please visit JIM website. Send your postal address by e-mail to jcim@163.com, we will send you a complimentary print issue upon receipt.

Editors-in-Chief: Wei-kang Zhao (China) \& Lixing Lao (USA). ISSN 2095-4964. Published by Science Press, China. 